\title{
Model Passengers' Travel Time for Conventional Bus Stop
}

\author{
Guangzhao Xin ${ }^{1}$ and Wei Wang ${ }^{2}$ \\ ${ }^{1}$ Jiangsu Key Laboratory of Urban ITS, Southeast University, Si Pai Lou No. 2, Nanjing 210096, China \\ 2 Jiangsu Province Collaborative Innovation Center of Modern Urban Traffic Technologies, Si Pai Lou No. 2, Nanjing 210096, China
}

Correspondence should be addressed to Guangzhao Xin; guangzhaoxin@seu.edu.cn

Received 12 November 2013; Revised 24 February 2014; Accepted 16 March 2014; Published 8 April 2014

Academic Editor: Tin-Tai Chow

Copyright (C) 2014 G. Xin and W. Wang. This is an open access article distributed under the Creative Commons Attribution License, which permits unrestricted use, distribution, and reproduction in any medium, provided the original work is properly cited.

\begin{abstract}
Limited number of berths can result in a subsequent bus stopping at the upstream of a bus stop when all berths are occupied. When this traffic phenomenon occurs, passengers waiting on the platform usually prefer walking to the stopped bus, which leads to additional walking time before boarding the bus. Therefore, passengers' travel time consumed at a bus stop is divided into waiting time, additional walking time, and boarding time. This paper proposed a mathematical model for analyzing passengers' travel time at conventional bus stop based on theory of stochastic service system. Field-measured and simulated data were designated to demonstrate the effectiveness of the proposed model. By analyzing the results, conclusion was conducted that short headway can reduce passengers' waiting time at bus stop. Meanwhile, the theoretical analysis explained the inefficiency of bus stops with more than three berths from the perspective of passengers' additional walking time. Additional walking time will increase in a large scale when the number of berths at a bus stop exceedsthe threshold of three.
\end{abstract}

\section{Introduction}

Bus stop is the connection between transit passenger and bus service system. Both the transit system and passenger satisfaction are under the influence of bus stop [1]. Travel time, as one of the most crucial factors determining the perception of transit service of quality, has been researched from various perspectives. Yetiskul and Senbil quantified the underlying determinants of public bus transit travel-time variability using data collected in Turkey's capital of Ankara [2]. Yu et al. adopted methods of support vector machine, artificial neural network, $k$ nearest neighbours algorithm, and linear regression to predict the travel time of multiple routes based on the real-world data in Hong Kong [3]. Abkowitz and Engelstein conducted analysis on the factors that influence the travel time of transit routes [4]. Strategies to decrease the travel time between bus stops were also studied by a number of researchers. Existing researches suggest that bus stop-skipping service can reduce vehicles' running time in a large scale for both the new stop-skipping service and the regular service running in parallel [5-7].

Besides the research on travel time on route level, a number of researchers have concentrated on the stop-level travel time. Guenthner and Hamat studied the effect of complicated fare structure on dwell time [8]. Levine and Torng made detailed regression analysis to identify the low floor's influence on dwell time [9]. In recent years, a number of stoplevel researches involved passengers' behavior in transport hubs, of which the waiting time at bus stops is one of the most widespread concerns. For example, Marguier and Ceder formulated the waiting time at bus stops of overlapping routes, where some routes are sharing common stops [10]. Chang and Hsu analyzed the passenger waiting time of an intercity transit station with feeder bus services and quantified the relations between passenger waiting time of intercity transit system and the reliability of feeder bus services [11]. Ohmori et al. examined passengers' waiting behaviour at bus stops and relationships between waiting behaviour, environment of bus stops, and passengers' attitudes and preferences towards bus stops [12]. The review of past researches on waiting time at bus stops can be classified into three categories. The first category studied the waiting time using microscopic simulation models $[13,14]$. The second involved studies that compared the actual waiting time with the time perceived by passengers $[15,16]$. In the third category, waiting time at bus 
stops was estimated based on the vehicles' arrival information $[17,18]$.

Using theory of classical queueing, the waiting time can be deduced based on the distribution of arrival headway and service time, as well as the number of service counter [19]. However, in some practical queueing systems, the service process can be discontinuous such as buses loading passengers. The boarding activity starts only when buses dwell in the scheduled berth and ends during the headway between two adjacent buses. Instead of classical queueing models, vacation queueing models can be applied to estimate the average waiting time at bus stops [20]. Limited number of berths can result in a subsequent bus vehicle stopping at the upstream of a bus stop when all berths are unavailable. Thus passengers waiting on the platform have to walk to the stopped bus. Additional walking time deduced from theory of probability and queueing was proposed for the first time in this paper to consider this situation. Therefore, passengers' travel time consumed at a bus stop is divided into waiting time, additional walking time, and boarding time. This paper firstly analyzes the traffic characteristics of bus and pedestrian at bus stops. Then a mathematical model for analyzing passengers' travel time at bus stops based on theory of stochastic service system was proposed. Next, field data was collected and simulation model was developed to identify the effectiveness of the proposed model. Finally, sensitivity analysis and conclusion were conducted.

\section{Traffic Characteristics at Bus Stop}

We make the assumption that passengers of bus route $i$ arrive at the scheduled bus stop at random. Passengers subsequently join the queueing team on the specified platform waiting for the bus's arrival. Figure 1 illustrates passengers' waiting process on platform at bus stop.

When approaching a bus stop, the bus usually decelerates to dwell in a berth at the bus stop. It takes approximately $9 \mathrm{~s}$ for the bus to complete the whole deceleration process before entering the scheduled bus stops [21]. The berth is designed according to the length of bus. Meanwhile, the number of berths at a bus stop should be limited because more than three berths can result in inefficiency [22]. However, the limited number of berths can lead to a subsequent bus vehicle that has to stop at the upstream of bus stops if all berths are occupied. When this traffic phenomenon occurs, passengers waiting on the platform usually prefer walking to the stopped bus, which leads to additional walking time before boarding the vehicle. Therefore, additional walking time besides waiting time and boarding time should be under consideration when estimating the travel time consumed by passengers at a bus stop.

\section{Formulate Passengers' Travel Time at Bus Stop}

In this paper, passengers' travel time at bus stops is divided into three parts: waiting time on the platform, additional walking time, and boarding time. Let $T$ denote the average

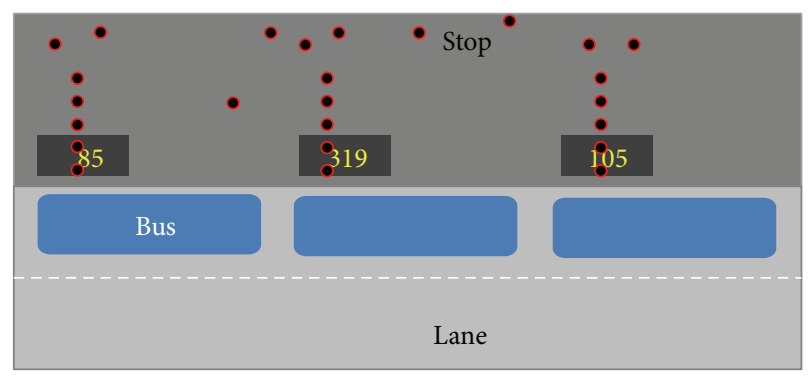

Figure 1: Passengers' waiting process at bus stop.

travel time consumed by passengers at a bus stop. The relationship can be written as

$$
T=t_{w}+t_{\alpha}+t_{b}
$$

where $t_{w}, t_{\alpha}$, and $t_{b}$ represent average waiting time, additional average walking time, and average boarding time, respectively.

3.1. Waiting Time. Theory of stochastic service system can be applied to conduct quantitative analysis for acquiring deep comprehension on the traffic mechanism introduced above. There exist two service processes, namely, buses entering berths and passengers boarding vehicles. In the former service process, a bus stop is the service counter. Similarly, buses are service counters when passengers are boarding vehicles. Based on queueing theory, the service time dwelling in a berth is assumed to obey negative exponential distribution. Buses and passengers arrive according to a Poisson process. It is worth noting that the service processes of vehicles entering berths and passengers boarding vehicles are different. When buses enter berths, the bus stop is always available. That is to say, the bus stop usually continues to be open to buses without prohibiting dwelling, which can be assumed as a classical queueing model. However, the practical process of serving passengers always becomes unavailable until the next vehicle comes. There exists absent service period due to headways between two adjacent buses. Classical queueing models cannot be applied to solve this problem. Instead, this case can be modeled with vacation queueing models. In the vacation queueing models, it allows servers to take vacations due to reasons such as taking a break and being checked for maintenance.

According to theory of vacation queueing, the waiting time in equilibrium-state can be divided into several independent random variables [20]. These variables are classified into two kinds, one of which is the waiting time derived from the corresponding classical queueing model without consideration of vacations and the rest of the parts are derivatives corresponding to vacation policies. Considering a classical queueing model and the corresponding vacation queueing model, let $W_{c}$ denote the waiting time of a classical queueing model in equilibrium-state. Correspondingly, $W_{v}$ represents the same performance in equilibrium-state of the 
corresponding vacation queueing model. The relationship among these variables can be formulated as

$$
W_{v}=W_{c}+W_{d}+W_{r}
$$

where $W_{d}$ and $W_{r}$ represent the derivative waiting time due to vacation policies.

In general, the concrete arithmetic expression cannot be deduced easily from the complicated Markov process. Probability generating function and Laplace transform are introduced to simplify these processes. Thus,

$$
W_{v}^{*}(s)=W_{c}^{*}(s) W_{d}^{*}(s) W_{r}^{*}(s),
$$

where $W^{*}(s)$ is the Laplace transform (LST) of the corresponding variables in equilibrium-state.

We make the assumption that passengers of bus route $i$ arrive at the scheduled bus stop according to a Poisson process with rate $\lambda_{p}^{i}$. Passengers subsequently join the queueing team on the specified platform waiting for the bus's arrival. That is to say, they comply with first-in-first-out (FIFO) queue discipline. When vehicles of bus route $i$ arrive in the berth, process of serving passengers for boarding occurs with a maximum threshold of design capacity. Different from the arrival process of passengers, boarding time in the service process is assumed to obey a general distribution, denoted by $B(t)$. Let

$$
\begin{gathered}
\frac{1}{\mu_{p}^{i}}=\int_{0}^{\infty} t d B(t), \quad b^{(2)}=\int_{0}^{\infty} t^{2} d B(t), \\
B^{*}(s)=\int_{0}^{\infty} e^{-s t} d B(t) .
\end{gathered}
$$

Obviously, passengers are not always being served by buses unless vehicles have pulled into the scheduled berths. The waiting time of passengers on the platform is different from that in a classical queueing system. There exists absent service period when process of serving passengers becomes unavailable due to headway between two adjacent buses. Classical queueing models cannot be applied to estimate the average waiting time. Instead, this case can be modeled with the vacation queueing models. Regarding headways between two adjacent vehicles as vacations taken by servicers, the vacation queueing model has vacation process besides arrival process, service process, and queue process in the classical queueing system. The vacation policy is determined by the traffic characteristics of vehicles' headways. To reflect the actual vacation process accurately, the following assumptions were made during the modeling process to estimate the average waiting time of passengers on the platform: (i) the moment when the door of a bus vehicle opens is the starting time of service process; (ii) the achievement of serving the last passenger is considered as the starting time of vacation process; (iii) vacation duration follows negative exponential distribution on the basis of Poisson process of vehicle's arrival.

Limited dwell time and design capacity of vehicles result in the fact that the number of passengers loaded during the dwell time cannot exceed the maximum threshold $M$. The stopped vehicle departures when either (i) $M$ passengers have been loaded or (ii) the queue becomes empty. Research by Takagi [23] was used to model the average waiting time of passengers on the platform. Choosing the moment when the door of a bus opens and the achievement instants of serving the last passenger as Markov embedded points, the Laplace transform of $W_{d}$ and $W_{r}$ is deduced as

$$
\begin{gathered}
W_{d}^{*}\left(\lambda_{p}^{i}(1-z)\right)=\frac{1-V^{*}\left(\lambda_{p}^{i}(1-z)\right)}{E(V) \lambda_{p}^{i}(1-z)}, \\
W_{r}^{*}\left(\lambda_{p}^{i}(1-z)\right)=L_{r}(z),
\end{gathered}
$$

where $V(x)$ denotes the headway duration, of which the Laplace transform is $V^{*}(x) . L_{r}(z)$ represents the probability generating function of additional queue length due to vacation effect. Let $q_{k}$ be the joint probability that the embedded point is starting serving passengers when $k$ passengers are present on the platform at this instant, and let $h_{j 0}$ denote the joint probability that the embedded point is the $j$ th serving passengers completion instant in a service period and that no passengers are present on the platform at this instant. By introducing $H_{0}(z)=\sum_{j=1}^{M-1} h_{j 0} z^{M-j}$, expression of $L_{r}(z)$ has been achieved as

$$
\begin{aligned}
L_{r}(z)= & \frac{1-\rho_{p}+\lambda_{p}^{i} E(V)}{1-\rho_{p}} z^{M} \\
& \times\left(\left(1-\left[\frac{B^{*}\left(\lambda_{p}^{i}(1-z)\right)}{z}\right]^{M}\right) q_{0}\right. \\
& \left.+H_{0}(1)+H_{0}\left[\frac{B^{*}\left(\lambda_{p}^{i}(1-z)\right)}{z}\right]\right) \\
& \times\left(z^{M}-V^{*}\left(\lambda_{p}^{i}(1-z)\right)\right. \\
& \left.\times\left(B^{*}\left(\lambda_{p}^{i}(1-z)\right)\right)^{M}\right)^{-1}
\end{aligned}
$$

where $\rho_{p}=\lambda_{p}^{i} / \mu_{p}^{i}$ is called traffic intensity and represents the average number of passengers during the boarding time of a passenger.

The average waiting time of passengers on the platform, $t_{w}$, can be obtained as

$$
t_{w}=E\left(W_{v}\right)=\frac{\lambda_{p}^{i} b^{(2)}}{2\left(1-\rho_{p}\right)}+\frac{E\left(V^{2}\right)}{2 E(V)}-E(V)+\frac{q^{\prime}(1)}{\lambda_{p}^{i} q(1)},
$$


where

$$
\begin{gathered}
q(1)=\frac{1-\rho_{p}}{1-\rho_{p}+\lambda_{p}^{i} E(V)}, \\
q^{\prime}(1)=\left(\lambda_{p}^{i} E(V)+M\right) q(1)-\frac{2\left(1-\rho_{p}\right)+\left(\lambda_{p}^{i}\right)^{2} b^{(2)}}{2\left(1-\rho_{p}+\lambda_{p}^{i} E(V)\right)} \\
-\frac{\left(1-\rho_{p}\right)^{2}\left[M(M-1) q_{0}+H_{0}^{\prime \prime}(1)\right]}{2\left[M\left(1-\rho_{p}\right)-\lambda_{p}^{i} E(V)\right]} \\
+\left(q ( 1 ) \left[\left(\lambda_{p}^{i}\right)^{2} M b^{(2)}-M(M-1)\left(1-\rho_{p}\right)^{2}\right.\right. \\
\left.\left.+\left(\lambda_{p}^{i}\right)^{2} E\left(V^{2}\right)+2 M \lambda_{p}^{i} \rho_{p} E(V)\right]\right) \\
\times\left(2\left[M\left(1-\rho_{p}\right)-\lambda_{p}^{i} E(V)\right]\right)^{-1} .
\end{gathered}
$$

Consider that the bus vehicles arrive with rate $\lambda_{b}^{i}$ and the dwell time in the berth is assumed as service time, which obeys negative exponential distribution with mean $1 / \mu_{b}^{i}$.

The average number of passengers loaded at the bus stop is

$$
Q=\frac{\lambda_{p}^{i}}{\lambda_{b}^{i}}=\frac{\mu_{P}^{i}}{\mu_{b}^{i}} .
$$

In this paper, the distribution of $h_{j 0}$ is estimated as

$$
h_{j 0}= \begin{cases}\frac{1 / j}{\sum_{j=1}^{Q} 1 / j}, & 1 \leq j \leq Q, \\ 0, & Q \leq j \leq M .\end{cases}
$$

3.2. Additional Walking Time. Limited number of berths can result in a subsequent bus vehicle stopping at the upstream of a bus stop if all berths are occupied. When this traffic phenomenon occurs, passengers waiting on the platform usually prefer walking to the stopped bus, which leads to additional walking time before boarding bus vehicle. Additional walking time consumed by passengers can be derived by dividing additional walking distance by passengers' walking speed. Thus, the average additional walking time can be calculated as

$$
t_{\alpha}=\frac{d_{\alpha}}{v_{p}}
$$

where $d_{\alpha}$ and $v_{p}$ denote average additional walking distance and walking speed of passengers.

This paper deduces the average additional walking distance, $d_{\alpha}$, based on theory of probability and queueing. Consider a bus steam with arrival rate $\lambda_{b}$. Buses pull into the scheduled stop with $m$ berths. Overtaking is prohibited when buses approach stops. Therefore, buses follow the firstin-first-out (FIFO) queueing discipline. The dwell time in the berth is assumed as service time, which obeys negative exponential distribution with mean $1 / \mu_{b}$. Let $l(t)$ denote the number of buses dwelling at the stop at time $t$. If $l(t) \leq m$, the subsequent coming vehicle can be served simultaneously by dwelling in the unoccupied berth. However, the subsequent coming vehicle will stop at the upstream of the scheduled bus stop when no berth is available to dwell in. According to the $M / M / m$ queueing model, the equilibrium-state distribution of $l(t)$ is

$$
\begin{gathered}
\pi_{0}=\left[\sum_{r=0}^{m-1} \frac{a^{r}}{r !}+\frac{a^{m}}{m !} \frac{1}{1-\rho_{b}}\right]^{-1}, \\
\pi_{r}= \begin{cases}\frac{\alpha^{r}}{r !} \pi_{0}, & r=1,2, \ldots, m, \\
\rho_{b}^{r-m} \pi_{m}, & r=m+1, m+2, \ldots,\end{cases}
\end{gathered}
$$

where $\alpha=\lambda_{b} / \mu_{b}$ and $\rho_{b}=\alpha / m$.

The probability that a bus stops at the upstream of the scheduled bus stop is

$$
P(l(t) \geq m)=\sum_{r=0}^{\infty} \pi_{r+m}=\frac{\pi_{m}}{1-\rho_{b}}=\frac{\alpha^{m}}{m !} \frac{m}{m-\alpha} \pi_{0},
$$

which means that the additional walking activity happens with the probability of $\pi_{m} /\left(1-\rho_{b}\right)$. Further, let $L$ and $h_{s}$ denote length of a bus and space headway between stopped vehicles. Then the average additional walking distance can be calculated as

$$
\begin{aligned}
d_{\alpha} & =\sum_{r=m}^{\infty} P(l(t)=r)(r-m+1)\left(L+h_{s}\right) \\
& =\sum_{i=0}^{\infty} \frac{m^{m} \rho^{m+i} \pi_{0}}{m !}(i+1)\left(L+h_{s}\right) \\
& =\frac{\left(m \rho_{b}\right)^{m}\left(L+h_{s}\right) \pi_{0}}{\left(1-\rho_{b}\right)^{2} m !} .
\end{aligned}
$$

Then the average additional walking time can be calculated as

$$
t_{\alpha}=\frac{\left(m \rho_{b}\right)^{m}\left(L+h_{s}\right) \pi_{0}}{v_{p}\left(1-\rho_{b}\right)^{2} m !} .
$$

3.3. Per Passenger Boarding Time. The boarding time per passenger is under the influence of a number of factors [24], of which vehicle types, in-vehicle congestion, and fare payment procedures are the most prominent. Low-floor vehicles which can decrease necessary steps to get on the bus can reduce the time required to serve each passenger. High in-vehicle congestion rate has a negative effect on the boarding time per passenger as it takes additional time for the passengers in vehicle to move to the back of the bus in order to make room for the boarding passengers. The average time to pay a fare also has significant impact on the boarding time per passenger. For example, proof-of-payment fare collection can shorten the boarding time per passenger. The practical per passenger boarding time, $t_{b}$, can be given as

$$
t_{b}=\frac{1}{\mu_{p}^{i}}=\int_{0}^{\infty} t d B(t) .
$$


TABLE 1: Selected bus stops to collect field data.

\begin{tabular}{cccccc}
\hline Type & Site & Stop & Direction & $N^{\mathrm{a}}$ & $L^{\mathrm{b}}$ \\
\hline \multirow{6}{*}{ Online } & 1 & Yuquan Station & WE & 2 & 4 \\
& 2 & Fuzhuo Station & NS & 2 & 5 \\
& 3 & Hunan Station & WE & 3 & 6 \\
& 4 & Daxinggong Station & NS & 3 & 7 \\
& 5 & Jimingsi Station & EW & 4 & 7 \\
& 6 & Taipingmen Station & WE & 4 & 8 \\
\hline \multirow{6}{*}{ Offline } & 7 & Fuzimiao Station & WE & 2 & 7 \\
& 8 & Zongtongfu Station & EW & 2 & 7 \\
& 9 & Beijing Dong Station & WE & 3 & 7 \\
& 10 & Xuanwuhu station & NS & 3 & 8 \\
& 11 & Gulou Station & SN & 4 & 9 \\
& 12 & Xinjiwkou station & NS & 4 & 10 \\
\hline
\end{tabular}

Note: NS and WE mean from north to south and from west to east, respectively.

${ }^{\mathrm{a}} N$ : number of design berths at the selected bus stop.

${ }^{\mathrm{b}} L$ : number of scheduled bus lines dwelling at the selected bus stops.

\section{Model Validation}

4.1. Data Collection. The proposed travel time model at bus stop was validated against both field-measured and simulated data. The field-measured data was collected from 12 bus stops in Nanjing of China. Cross-sectional analysis was conducted to compare the field data collected at bus stops with different number of berths. To ensure that the traffic and geometric characteristics at different types of bus stops were similar, the following criteria were applied during bus stop selection: (i) the selected bus stops are separated from bicycle lane to avoid the influence of bicycle stream; (ii) no access is located adjacent to the selected bus stops; (iii) distance from selected bus stops to the adjacent intersections is larger than $150 \mathrm{~m}$; (iv) the selected bus stops have a larger boarding demand than alighting demand in the p.m. peak period; (v) exclude major transfer stops with large interchanging passenger demand.

Geometric characteristics of selected bus stops are shown in Table 1. Of the selected bus stations, six are online stops, and the others are offline stops. Bus stops with only one berth are usually located by occupying the bicycle lane, which are under the influence of bicycle stream. Therefore, this paper does not take into consideration the bus stops with one berth to maintain consistency in the analyzed bus stops. Video cameras were applied to record traffic data. A total of $30 \mathrm{~h}$ of traffic data at the selected bus stops had been collected in the weekday p.m. peak period, which is reviewed in the laboratory by the survey team to obtain the following information: (i) the time at which each bus vehicle pulled in and out of stops; (ii) the time at which the boarding activity began and ended; (iii) the number of passengers each bus vehicle loaded; (iv) the time at which passengers joined the queue waiting for buses; (v) the additional walking time consumed by passengers in the queue when bus vehicle stopped at the upstream of bus stops.

The simulated data was generated from the simulation models developed for all the selected bus stops. A widely used microscopic simulation package VISSIM 5.4 was used in this study to develop simulation models for the selected bus stops. To more accurately reflect actual traffic characteristics at the selected bus stops, several issues should be considered seriously: (i) parameters of traffic facilities such as public transit stops, platform, and vehicles in the simulation models were defined according to the real condition; (ii) multiple waiting area of platform was set to simulate the actual queueing phenomenon, and routing decisions for passengers were located in the area of the platform edge; (iii) pedestrian inputs were added as an origin for boarding passengers according to the statistical data; (iv) define start and destination areas for passengers of each bus route to measure the travel time at bus stops.

4.2. Calibration of Travel Time Model at Bus Stop. The travel time at bus stop estimated with the proposed model in this paper was compared with the data measured in the field and simulation models. The mean absolute percent error (MAPE) was applied to compare the differences among the estimated, field-measured, and simulated travel time at selected bus stops. The MAPE value can be obtained as

$$
\text { MAPE }=\frac{1}{n} \sum_{j=1}^{n}\left|\frac{t_{e}^{j}-t^{j}}{t^{j}}\right|,
$$

where MAPE is the mean absolute percent error between the estimated and field-measured or simulated average travel time at selected bus stops; $n$ represents the number of selected bus stops; $t_{e}^{j}$ denotes the estimated average travel time for bus stop $j$; and $t^{j}$ denotes the field-measured or simulated average travel time for bus stop $j$.

The MAPE values between the estimated and fieldmeasured or simulated average travel time of $i$ th passenger in the queue are illustrated in Figure 2. The estimated MAPE values vary from $1.86 \%$ to $85.39 \%$. The average MAPE value between the estimated and the measured travel time of the third passenger in the queue is 3.39\%. The average MAPE value between the estimated and the simulated travel time of the third passenger in the queue is $5.52 \%$. The average MAPE value between the estimated and the measured or simulated travel time of the third passenger in the queue is $4.45 \%$. Thus the travel time of third passenger in the queue can demonstrate the effectiveness of the proposed model in this paper.

\section{Sensitivity Analysis}

With the proposed model estimating passengers' travel time at bus stops, sensitivity analyses were conducted to identify the effects of crucial parameters on the passengers' travel time at bus stops.

The effect of arrival rate on the average waiting time is illustrated in Figure 3. The average waiting time $\left(W_{v}\right)$ decreases with an increase in the arrival rate of buses. Further, this decrease mainly results from the large-scale decline in the waiting time due to vacation policy $\left(W_{d}\right)$ considering the visualized fact shown in the same figure that the classical 


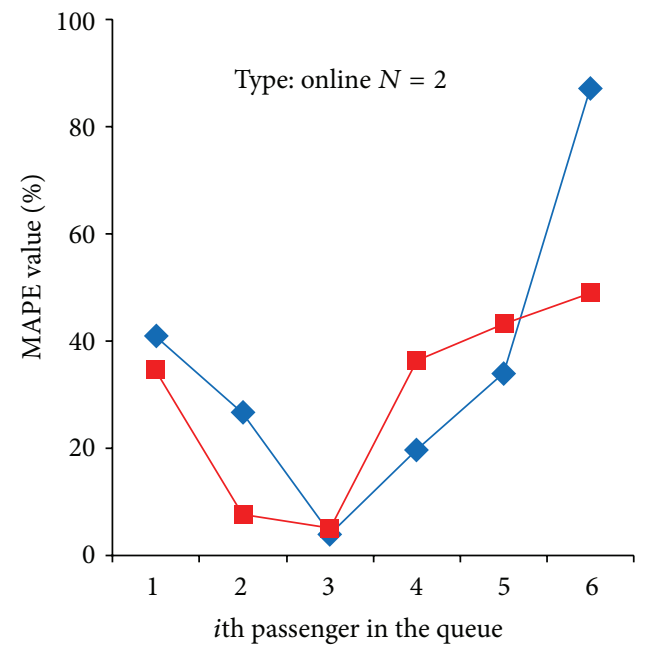

(a)

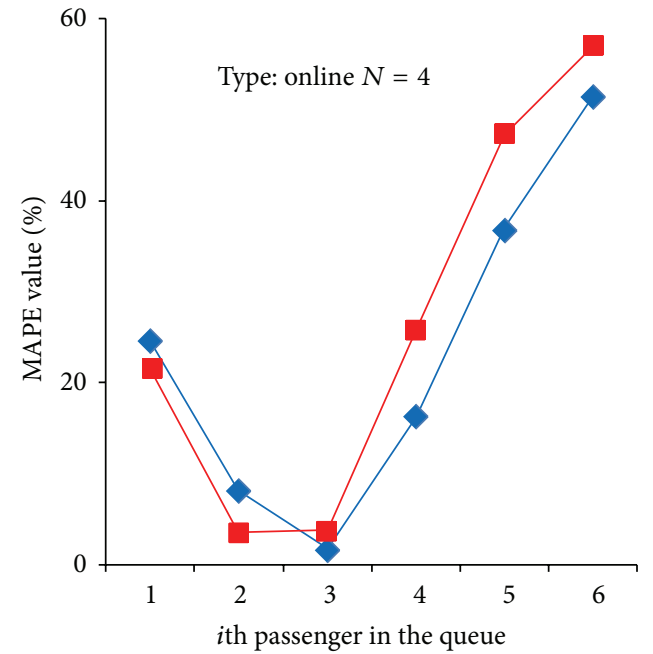

(c)

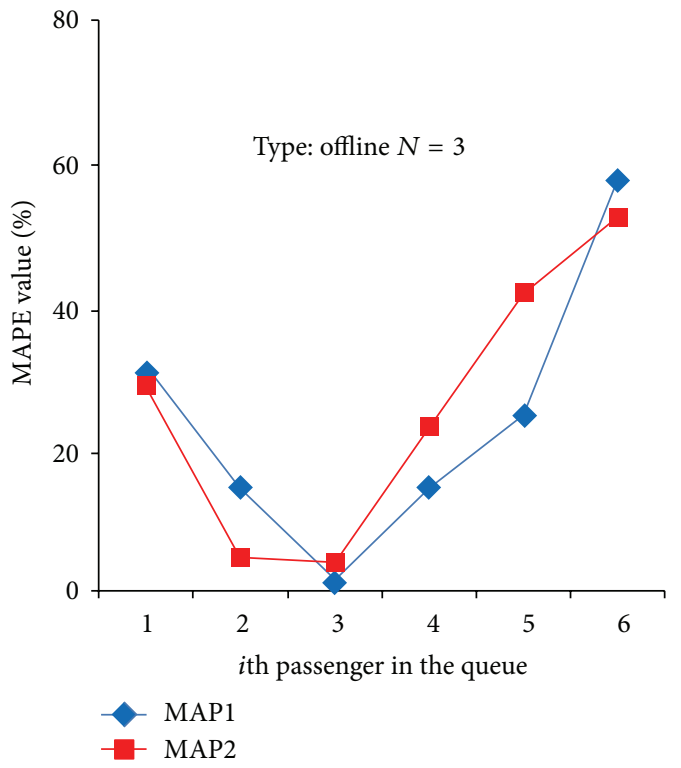

(e)

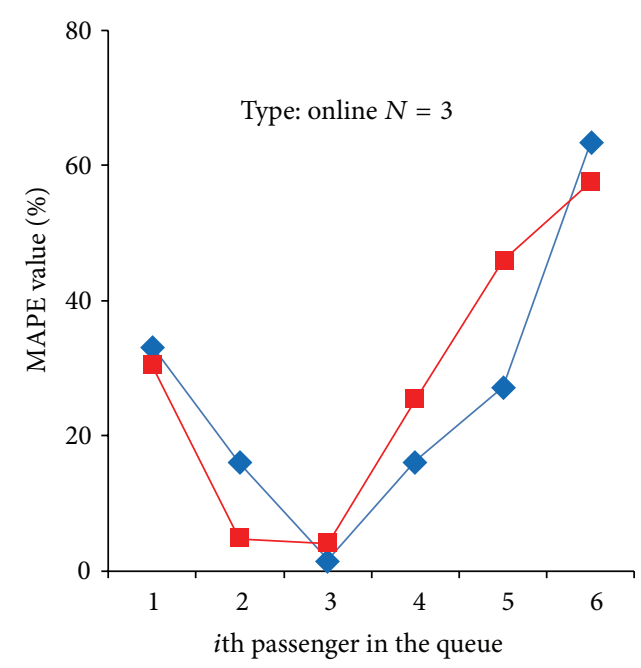

(b)

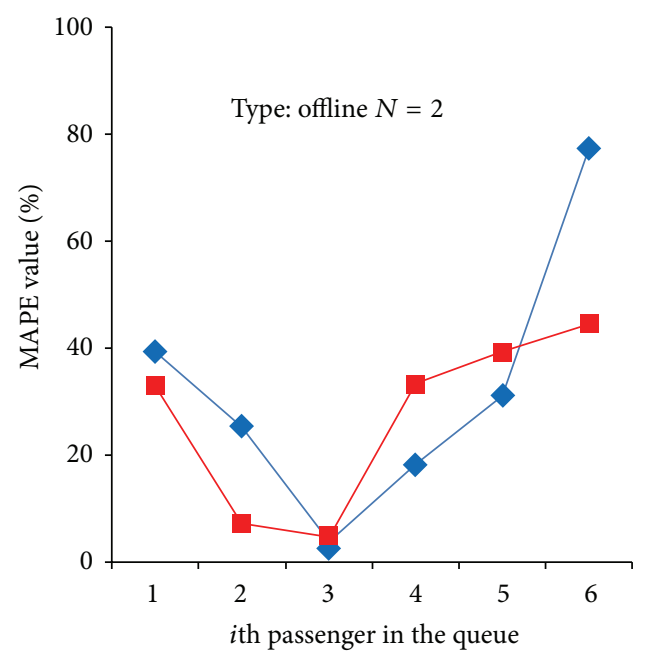

(d)

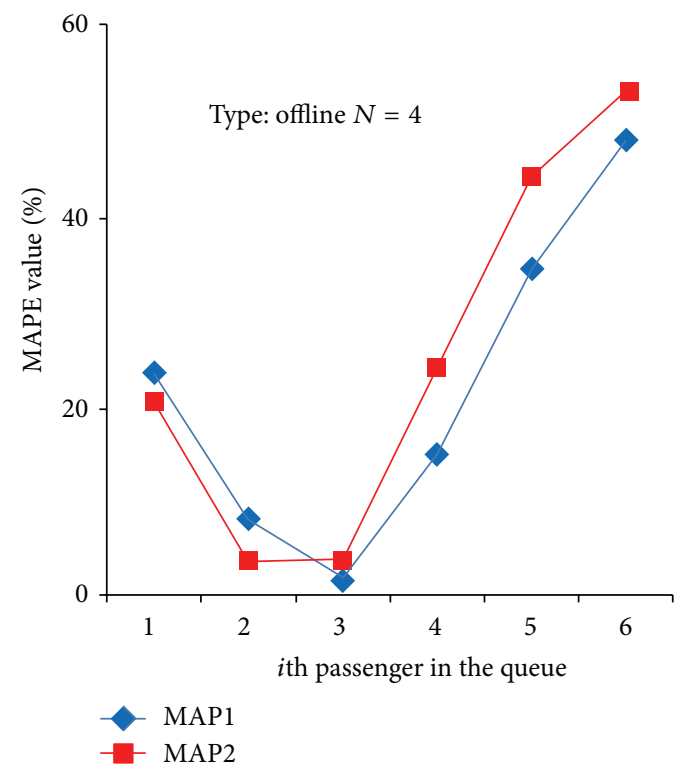

(f)

FIGURE 2: The MAPE value between the estimated and field-measured or simulated average travel time of $i$ th passenger in the queue. 


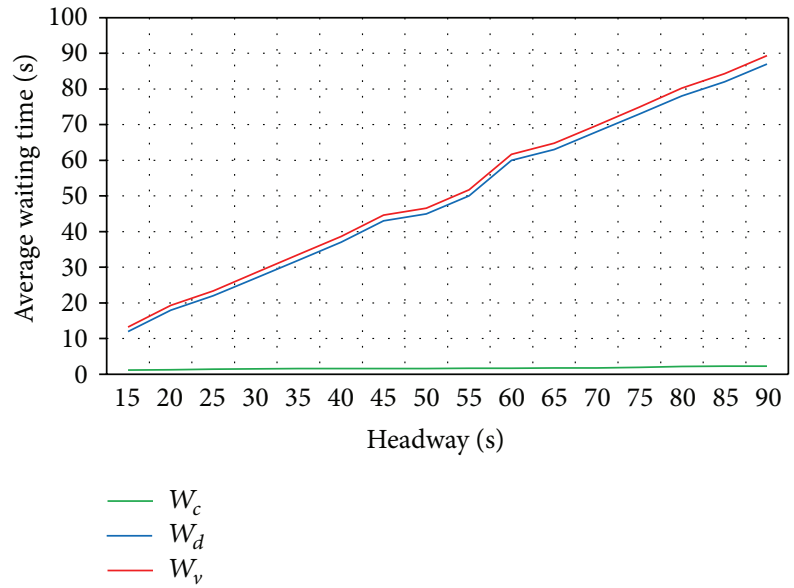

Figure 3: Average waiting time with different arrival rate of buses.

waiting time $\left(W_{c}\right)$ is insensitive to changes in the arrival rate of buses. This suggests that increase in the departure frequency of buses can result in the improvement of the average waiting time.

In Figure 4, sensitivity analysis was conducted subsequently to identify the effects of dwell time and number of berths on the additional walking time. The additional walking time increases with the increase in the number of berths at bus stops, whose increasing tendency is more obvious when the number of berths is larger than three. This suggests that it is preferable to design not more than three berths at bus stops to decrease the additional walking time. The effect of arrival rate on additional walking time is shown in Figure 5. The additional walking time increases with the increase of headway. When the headway is $100 \mathrm{~s}$, the additional walking time of a four-berth bus stop is approximately $94 \mathrm{~s}$, nearly five times as large as a two-berth bus stop. This once more suggests that more than three berths at a bus stop can result in the increase of additional walking time in a large scale, which should be considered seriously in the process of designing a bus stop.

\section{Conclusion}

Passengers' travel time at bus stops is modeled based on the theory of queueing and probability. The proposed model divides the travel time at bus stops into three parts: waiting time on the platform, additional walking time, and boarding time. Field-measured and simulated data were obtained to demonstrate the effectiveness of the proposed model. Analysis was conducted to identify the effects of crucial parameters such as arrival rate of buses, dwell time, and number of berths at the selected bus stops on passengers' travel time at bus stops. Based on the results of data analysis, the following conclusion can be conducted.

The waiting time on the platform results mainly from the unavailable service period during headways between two adjacent buses. The service period does not significantly affect the waiting time. Therefore, the emphasis of strategy to

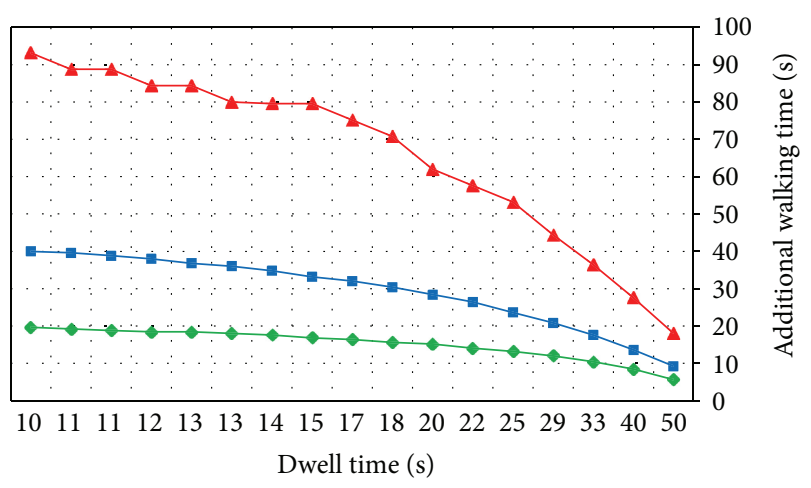

$\begin{aligned} \rightarrow m & =2 \\ -m & =3 \\ \rightarrow m & =4\end{aligned}$

FIgUre 4: Additional walking time at different dwell times for various berths, $\lambda_{b}=0.025 \mathrm{veh} / \mathrm{s}$.

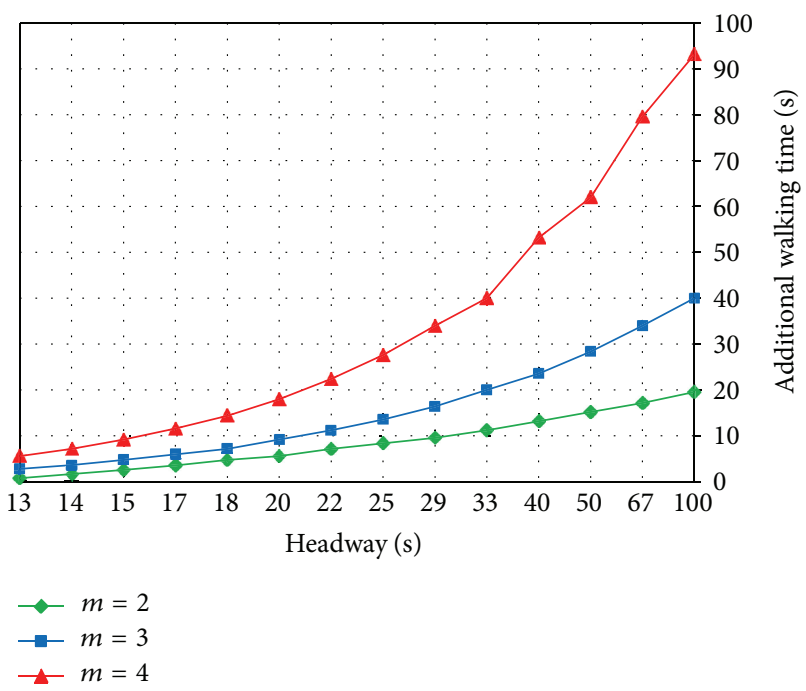

Figure 5: Additional walking time at different arrival rate of buses for various berths, $\mu_{b}=0.04 \mathrm{veh} / \mathrm{s}$.

decrease the waiting time should be placed on the headways, not on the boarding time.

The additional walking activity occurs when no berth at the scheduled bus stop is available for the approaching bus. However, it does not mean that implementing more berths can eliminate the additional walking time. On the contrary, as analysis suggests, the average additional walking time increases in a large scale when the number of berths at a bus stop exceeds the threshold of three. Analysis also shows that the additional walking time increases with an increase in headways between two adjacent buses. Increase in dwell time can, however, lead to a decline in the additional walking time. The theoretical analysis explains the inefficiency of bus stops with more than three berths from the perspective of passengers' additional walking time, which is significant in the design of the bus stop. 
Bus stops are usually placed near signalized intersections. Further research is needed to identify the effect of signalized intersections on passengers' travel time at the near-side bus stops. Furth and SanClemente [21] and Guo et al. [25] researched the effect of signalized intersections on vehicles based on kinematic wave theory. With the effect of signalized intersections on buses, passengers' travel time at bus stops can be affected. Therefore, a new model for estimating passengers' travel time at near-side bus stops is needed in the future research.

\section{Notations}

$B(t)$ : General distribution of boarding time

$d_{\alpha}$ : $\quad$ Average additional walking distance

$h_{j 0}$ : The joint probability that the embedded point is the $j$ th serving passengers completion instant in a service period and that no passengers are present on the platform at this instant

$h_{s}$ : $\quad$ Space headway between stopped vehicles

$L: \quad$ Length of a bus

$l(t)$ : Number of buses dwelling at the stop at time $t$

$L_{r}(z)$ : The probability generating function of additional queue length due to vacation effect

M: $\quad$ The maximum threshold number of passengers loaded during the dwell time

$q_{k}$ : The joint probability that the embedded point is the starting of serving passengers and that $k$ passengers are present on the platform at this instant

$T: \quad$ Average travel time consumed by passengers at a bus stop

$t_{\alpha}: \quad$ Additional average walking time

$t_{b}$ : Average boarding time

$t_{w}$ : Average waiting time

$\mu_{p}^{i}: \quad$ The average service time per passenger

$v_{p}: \quad$ Walking speed of passengers

$V(x)$ : The headway duration

$V^{*}(x)$ : The Laplace transform of headway duration

$W_{c}$ : Waiting time of a classical queueing model in equilibrium-state

$W_{v}$ : Waiting time in equilibrium-state of the corresponding vacation queueing model

$W_{d}: \quad$ The derivative waiting time due to vacation policies

$W_{r}: \quad$ The derivative waiting time due to vacation policies

$W^{*}(s)$ : The Laplace transform of the corresponding variables in equilibrium-state

$\lambda_{p}^{i}: \quad$ The rate of Poisson process of passengers of bus route $i$ arriving at the scheduled bus stop

$\lambda_{b}^{i}: \quad$ Arrival rate of bus.

\section{Conflict of Interests}

The authors declare that there is no conflict of interests regarding the publication of this paper.

\section{Acknowledgments}

This research was sponsored by the Major State Basic Research Development Program of China (no. 2012CB725400) and the Key Project of National Natural Science Foundation of China (no. 51338003). The authors would like to thank the survey team from the School of Transportation in Southeast University for their assistance in collecting and processing field data.

\section{References}

[1] Federal Transit Administration, "Guidelines for the location and design of bus stops," Transit Cooperative Research Program 19, Transportation Research Board, Washington, DC, USA, 1996.

[2] E. Yetiskul and M. Senbil, "Public bus transit travel-time variability in Ankara (Turkey)," Transport Policy, vol. 23, pp. 5059, 2012.

[3] B. Yu, W. H. K. Lam, and M. L. Tam, "Bus arrival time prediction at bus stop with multiple routes," Transportation Research C: Emerging Technologies, vol. 19, no. 6, pp. 1157-1170, 2011.

[4] M. D. Abkowitz and I. Engelstein, "Factors affecting running time on transit routes," Transportation Research A: General, vol. 17, no. 2, pp. 107-113, 1983.

[5] Z. Y. Liu, Y. D. Yan, X. B. Qu, and Y. Zhang, "Bus stop-skipping scheme with random travel time," Transportation Research $C$, vol. 35, pp. 46-56, 2013.

[6] P. R. Tétreault and A. M. El-Geneidy, "Estimating bus run times for new limited-stop service using archived AVL and APC data," Transportation Research A: Policy and Practice, vol. 44, no. 6, pp. 390-402, 2010.

[7] E. I. Diab and A. M. El-Geneidy, "Understanding the impacts of a combination of service improvement strategies on bus running time and passenger's perception," Transportation Research A: Policy and Practice, vol. 46, no. 3, pp. 614-625, 2012.

[8] R. P. Guenthner and K. Hamat, "Transit dwell time under complex fare structure," Journal of Transportation Engineering, vol. 114, no. 3, pp. 367-379, 1988.

[9] J. C. Levine and G.-W. Torng, "Dwell-time effects of low-floor bus design," Journal of Transportation Engineering, vol. 120, no. 6, pp. 914-929, 1994.

[10] P. H. J. Marguier and A. Ceder, "Passenger waiting strategies for overlapping bus routes," Transportation Science, vol. 18, no. 3, pp. 207-230, 1984.

[11] S. K. J. Chang and C.-L. Hsu, "Modeling passenger waiting time for intermodal transit stations," Transportation Research Record, vol. 1753, pp. 69-75, 2001.

[12] N. Ohmori, T. Hirano, N. Harata, and K. Ohta, "Passengers' waiting behavior at bus stops," in Traffic and Transportation Studies: Proceedings of the 4th International Conference on Traffic and Transportation Studies (ICTTS '04), pp. 157-164, August 2004.

[13] R. Fernández, "Modelling public transport stops by microscopic simulation," Transportation Research C: Emerging Technologies, vol. 18, no. 6, pp. 856-868, 2010. 
[14] Q. Zhang and B. M. Han, "Simulation model of pedestrian interactive behavior," Physica A: Statistical Mechanics and its Applications, vol. 390, no. 4, pp. 636-646, 2011.

[15] D. B. Hess, J. Brown, and D. Shoup, "Waiting for the bus," Journal of Public Transportation, vol. 7, no. 4, pp. 67-84, 2004.

[16] I. Psarros, K. Kepaptsoglou, and M. G. Karlaftis, "An empirical investigation of passenger wait time perceptions using HazardBased duration models," Journal of Public Transportation, vol. 14, no. 3, pp. 109-122, 2011.

[17] P. G. Furth and T. H. J. Muller, "Service reliability and hidden waiting time insights from automatic vehicle location data," Transportation Research Record, vol. 1955, pp. 79-87, 2006.

[18] F. McLeod, "Estimating bus passenger waiting times from incomplete bus arrivals data," Journal of the Operational Research Society, vol. 58, no. 11, pp. 1518-1525, 2007.

[19] H. Kobayashi, B. L. Mark, and W. Turin, Probability, Random Processes, and Statistical Analysis, Cambridge University Press, Cambridge, New York, NY, USA, 2012.

[20] N. Tian and Z. G. Zhang, Vacation Queueing Models Theory and Applications, Springer, Boston, Mass, USA, 2006.

[21] P. G. Furth and J. L. SanClemente, "Near side, far side, uphill, downhill: impact of bus stop location on bus delay," Transportation Research Record, vol. 1971, pp. 66-73, 2006.

[22] National Research Council, Highway Capacity Manual, Transportation Research Board, Washington, DC, USA, 2000.

[23] H. Takagi, Queueing Analysis: Vacation and Priority Systems, North-Holland, Amsterdam, The Netherlands, 1993.

[24] Federal Transit Administration, "Transit capacity and quality of service manual," Transit Cooperative Research Program Report 100, Transportation Research Board, Washington, DC, USA, 2003.

[25] W. H. Guo, M. J. Cassidy, V. V. Gayah, and Y. F. Ouyang, "Mitigating negative impacts of near-side bus stops on cars," Transportation Research B, vol. 47, pp. 42-56, 2013. 


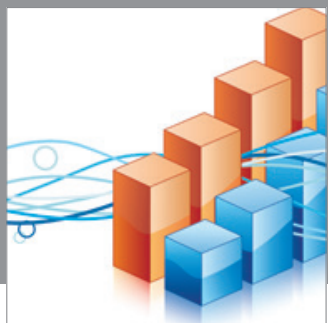

Advances in

Operations Research

mansans

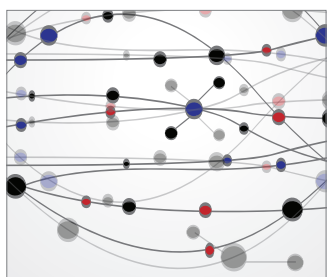

The Scientific World Journal
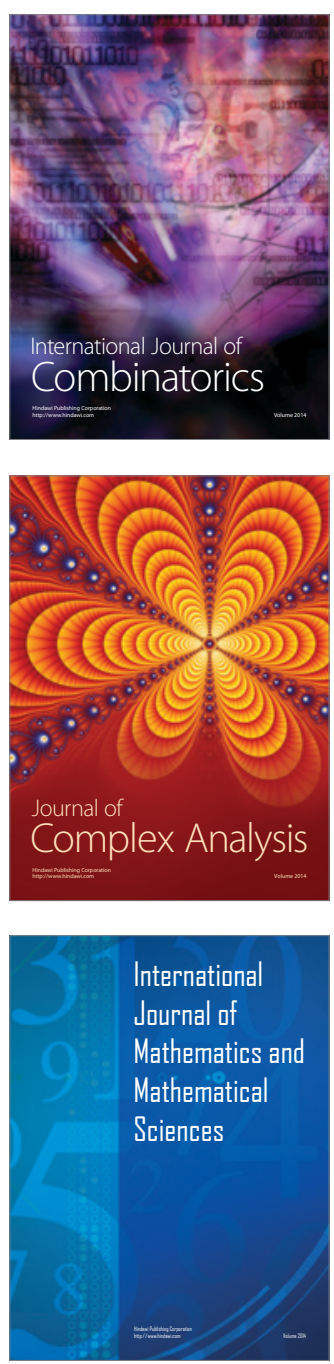
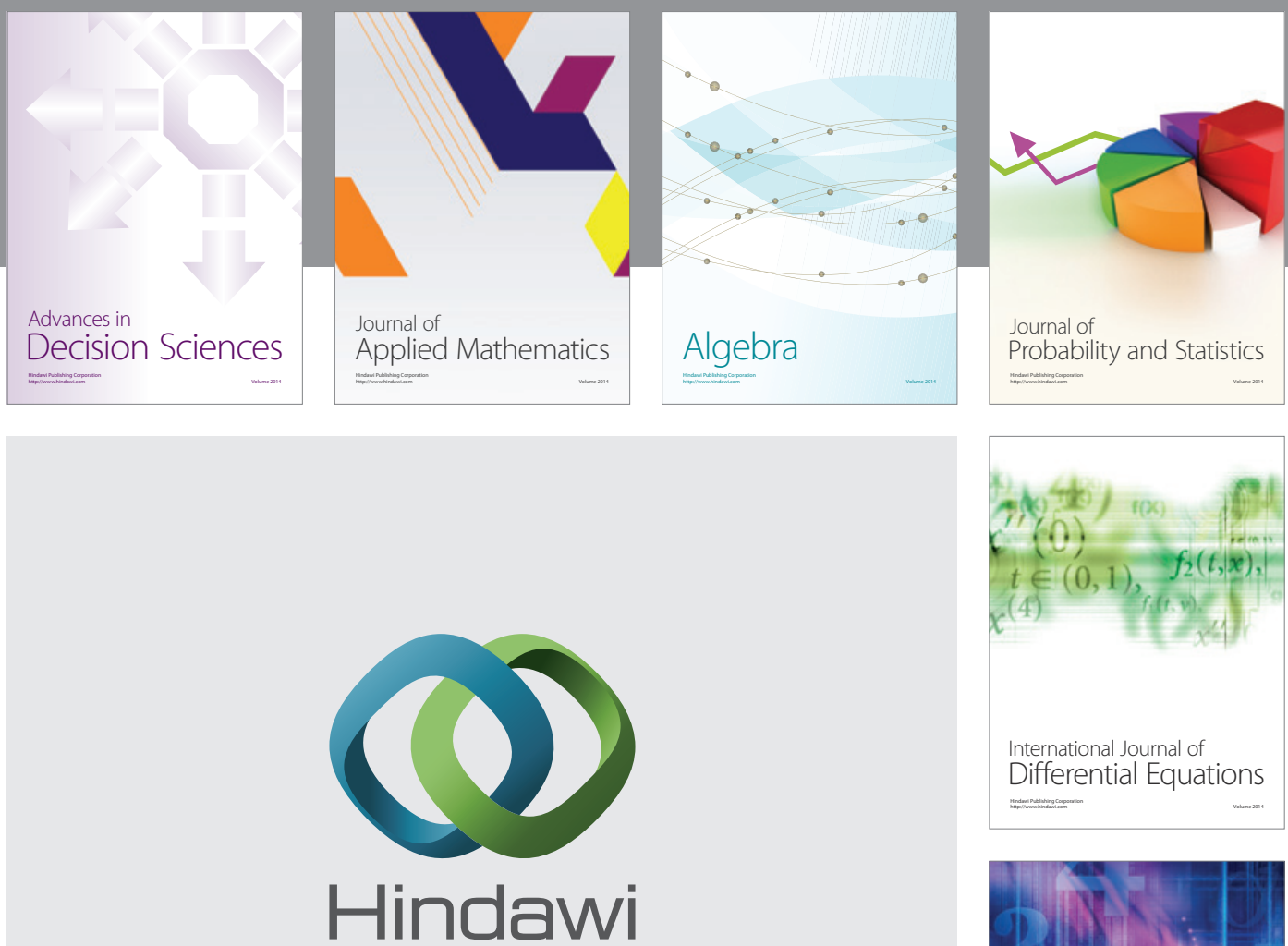

Submit your manuscripts at http://www.hindawi.com
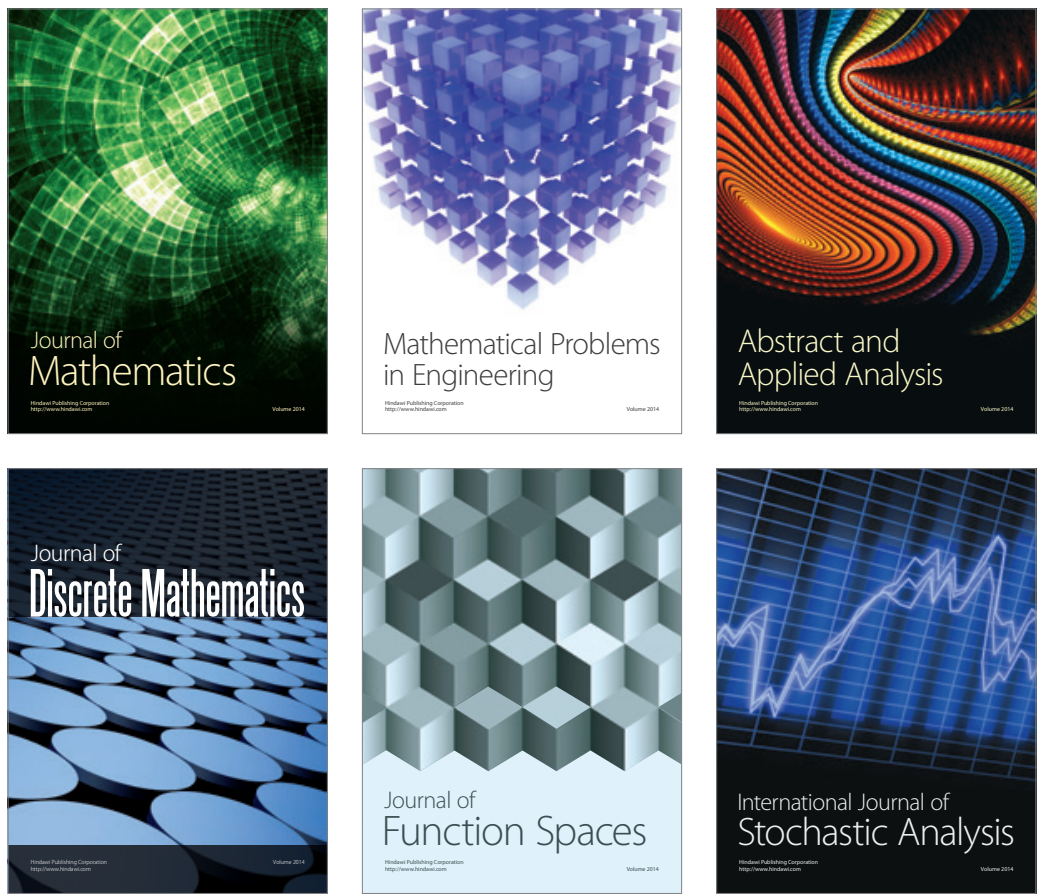

Journal of

Function Spaces

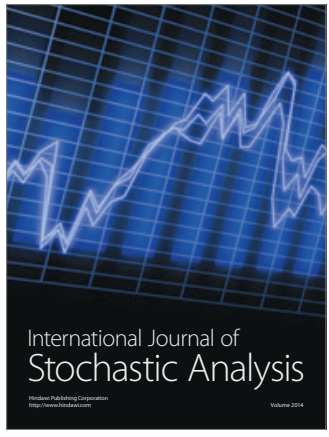

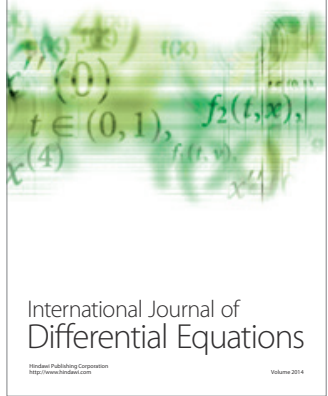
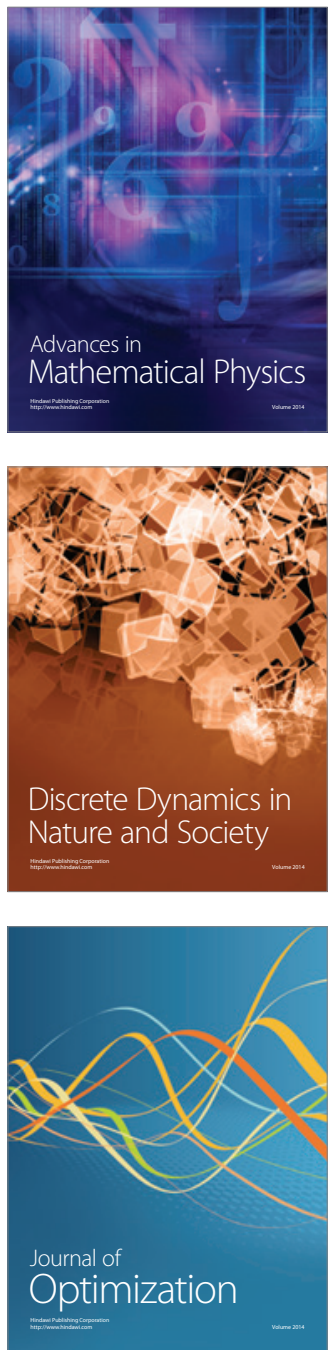